\title{
Market dynamics driven by the decision-making of both power producers and transmission owners
}

\author{
Anna Minoia, Member, IEEE \\ Politecnico di Bari \\ a.minoia@poliba.it
}

\author{
Damien Ernst, Member, IEEE \\ University of Liège \\ dernst@ulg.ac.be
}

\author{
Marija Ilic, Fellow, IEEE \\ Carnegie Mellon University \\ milic@ece.cmu.edu
}

\begin{abstract}
In this paper we consider an electricity market in which not only the power producers but also the transmission owners can submit a bid. The market is cleared at each stage by minimizing the sum of the production prices and the transmission prices.

A model of the strategic behavior is formulated for the different agents of the system. This strategic behavior modelling leads to a market dynamics that can be used to determine the different payoffs of the agents over a temporal horizon.

Simulations are carried out for several configurations of this two node power system. The influence of the transfer capacity and the market structure on the payoffs of the different agents is discussed.
\end{abstract}

Index Terms-Active transmission owner agent, competition modelling, market dynamics

\section{INTRODUCTION}

The transmission network is a key element in the electricity market. It allows producers and consumers to access the network and inject or withdraw the power contracted in the market. However, while competition has been introduced between producers and consumers, the owner of the transmission grid continues to have only a passive role in the market. The major reason for this choice was related to the idea of natural monopoly of the transmission network.

Nevertheless, recent market design proposals have analyzed the possibility of providing incentives for transmission investments, and of promoting, through performance settlement criteria, a more efficient usage of the transmission network [1]- [2].

Different types of schemes have been proposed based on the creation of short and long term markets for Financial Transmission Rights (FTR) [3], or for the regulation of prices of Independent Transmission Companies [4].

In this paper, we consider the Transmission Owner (TO) as an agent that can express its willingness to be paid for transmission service through a bid. We define a new market mechanism in which the objective function takes into account both the transmission bids and the power producer offers. To analyze what the different payoffs of the agents in this market structure may be, we model their behavior which leads to a market dynamics. The payoffs over a certain time horizon are computed by integrating this dynamics over this period of time.

The mathematical formulation is, for the sake of simplicity, related to a two node power system.

The paper is structured as follows. Section II explains the market characteristics and the strategy adopted to model the different agents. Section III details the simulation conditions while in Sections IV and V, simulation results related to different power systems are given and discussed. In Section VI we illustrate the influence of the market type on the different agents' rewards by comparing the previously computed results with the ones that would have been obtained with a traditional locational price market. And finally Section VII concludes.

\section{POWER SYSTEM DESCRIPTION}

We consider here a power system composed of two nodes $A$ and $B$. A load is connected at each node, load $A$ and load $B$. Each load has a constant inelastic demand of $q_{A}$ and $q_{B}$. The line that connects these two nodes is supposed to be a loss-less transmission line, which can only transfer a limited amount of power $(K)$. Generators are connected at each end of the line. Figure 1 represents the two node power system considered.

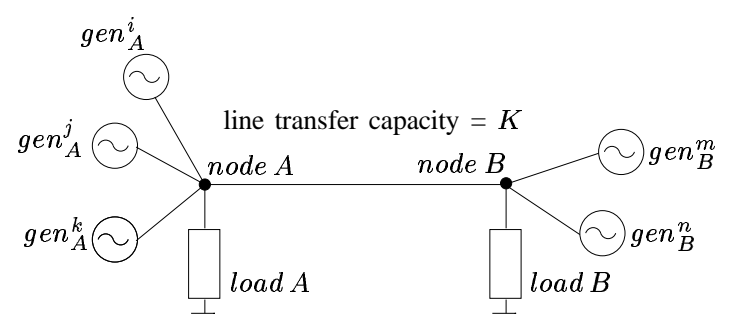

Fig. 1. A simple power system composed of generators and loads connected through a single transmission line.

We suppose that the active market participants are the generators and the TO. Moreover, we suppose that the marginal cost of each generator is constant and that each of them can only produce a finite amount of power.

Let $\operatorname{gen}_{A}^{1}, \operatorname{gen}_{A}^{2}, \cdots, \operatorname{gen}_{A}^{n_{A}}\left(\operatorname{gen}_{B}^{1}, \operatorname{gen}_{B}^{2}, \cdots, \operatorname{gen}_{B}^{n_{B}}\right)$ be the $n_{A}\left(n_{B}\right)$ generators of the system connected to node $A(B)$. Let $c_{A}^{i}\left(c_{B}^{i}\right)$ be the marginal cost of generator $g e n_{A}^{i}$ $\left(\operatorname{gen}_{B}^{i}\right)$ and $q_{A_{\max }}^{i}\left(q_{B_{\max }}^{i}\right)$ the maximum amount of power it can produce. Moreover, let $T O$ be the transmission owner of the line.

We suppose that an Independent System Operator (ISO) is in charge of clearing the market. Furthermore, we refer to each session of the market as a stage. In the following the market clearing mechanism, the payoffs of the different agents and their bidding strategies are illustrated.

\section{A. The market clearing mechanism}

We suppose that each generator $g e n_{A}^{i}\left(\right.$ gen $\left._{B}^{i}\right)$ submits a bid $b_{A}^{i}\left(b_{B}^{i}\right)$ that represents the minimum price the generator $g e n_{A}^{i}$ $\left(\right.$ gen $\left._{B}^{i}\right)$ asks per MW to produce. Furthermore, we suppose that the $T O$ submits a bid $b_{T O}$ that represents the price the transmission owner asks per MW flowing through the line. 
The clearing mechanism for the market consists of finding which quantity of power each generator has to produce, and consequently how many MWs flow into the line, in order to satisfy the load demand at the minimum expenditure.

This mechanism can be stated as follows :

Determine $\left(q_{A}^{1}, \cdots, q_{A}^{n_{A}}, q_{B}^{1} \cdots, q_{B}^{n_{B}}\right) \in \mathbb{R}^{n_{A}+n_{B}}$ that minimizes

$$
\sum_{i=1}^{n_{A}} b_{A}^{i} q_{A}^{i}+\sum_{i=1}^{n_{B}} b_{B}^{i} q_{B}^{i}+b_{T O}\left|\sum_{i=1}^{n_{A}} q_{A}^{i}-q_{A}\right|
$$

under the following constraints

$$
\begin{array}{r}
\sum_{i=1}^{n_{A}} q_{A}^{i}+\sum_{i=1}^{n_{B}} q_{B}^{i}=q_{A}+q_{B} \\
\sum_{i=1}^{n_{A}} q_{A}^{i}-q_{A} \leq K, \quad \sum_{i=1}^{n_{B}} q_{B}^{i}-q_{B} \leq K \\
0 \leq q_{A}^{i} \leq q_{A_{\max }}^{i}, \quad 0 \leq q_{B}^{i} \leq q_{B_{\max }}^{i}
\end{array}
$$

Note that: $\left|\sum_{i=1}^{n_{A}} q_{A}^{i}-q_{A}\right|=\left|\sum_{i=1}^{n_{B}} q_{B}^{i}-q_{B}\right|$.

Once the problem is solved, i.e. once we have computed $\left(q_{A}^{1}, \cdots, q_{A}^{n_{A}}, q_{B}^{1} \cdots, q_{B}^{n_{B}}\right)$, the energy prices at node $A$ and $B\left(p_{A}\right.$ and $\left.p_{B}\right)$ are determined as follows ${ }^{1}$ :

$$
\begin{gathered}
p_{A}=\max \left(\max _{i=1, \cdots, n_{A}}\left(\mathbf{1}\left(q_{A}^{i} \neq 0\right) * b_{A}^{i}\right), \max _{i=1, \cdots, n_{B}}(\right. \\
\left.\left.\mathbf{1}\left(q_{B}^{i} \neq 0 \text { and } \sum_{i=1}^{n_{B}}\left(q_{B}^{i}-q_{B}\right) \geq 0\right) *\left(b_{B}^{i}+b_{T O}\right)\right)\right) \\
p_{B}=\max \left(\max _{i=1, \cdots, n_{B}}\left(\mathbf{1}\left(q_{B}^{i} \neq 0\right) * b_{B}^{i}\right), \max _{i=1, \cdots, n_{A}}(\right. \\
\left.\left.\mathbf{1}\left(q_{A}^{i} \neq 0 \text { and } \sum_{i=1}^{n_{A}}\left(q_{A}^{i}-q_{A}\right) \geq 0\right) *\left(b_{A}^{i}+b_{T O}\right)\right)\right)
\end{gathered}
$$

The idea is to establish a price for each node equal to the maximum between (i) the marginal price of the energy produced at that node and (ii) the sum of the marginal price of the energy produced somewhere else and the marginal price of transmission needed to deliver that energy to the node under analysis. It is useful to note that these nodal prices represent for the load a signal of energy and transmission price.

\section{B. The payoffs of the different agents}

Once the dispatched quantities are obtained and the prices for energy at the different nodes are computed, it is possible to determine the payoffs (or rewards) of the different agents of the power system. In particular, we assume that the nodal price is the price paid by loads and received by generators, at the bus. Whereas the $T O$ is paid at a price equal to its bid.

The mathematical formulation of the rewards is described below :

Power producer agent : The payoff of a power producer agent is equal to the money it gets for producing the electricity minus the costs of producing it. The payoff of the power producer agents $g e n_{A}^{i}$ and $g e n_{B}^{i}$ are :

$$
\begin{aligned}
& r_{g e n_{A}^{i}}=q_{A}^{i} *\left(p_{A}-c_{A}^{i}\right) \\
& r_{g e n_{B}^{i}}=q_{B}^{i} *\left(p_{B}-c_{B}^{i}\right)
\end{aligned}
$$

\footnotetext{
${ }^{1} \mathbf{1}($ logical_expression $)=1$ if logical_expression $=$ true and zero otherwise.
}

Transmission Owner agent : The payoff of the $T O$ is equal to the money that it gets for the MWs flowing in its line. The payoff of the $T O$ is :

$$
r_{T O}=b_{T O} *\left(\left|\sum_{i=1}^{n_{A}} q_{A}^{i}-q_{A}\right|\right)
$$

Note that since the absolute value of the flow is considered, the $T O$ is paid independently of the direction of the flow.

Load agents : We consider the payoffs of the load agents load $A$ and load $B$ are equal to "minus the money they pay for buying the electricity":

$$
\begin{aligned}
& r_{\text {load } A}=-q_{A} * p_{A} \\
& r_{\text {load } B}=-q_{B} * p_{B}
\end{aligned}
$$

It could happen that the total amount of money paid by the customers is greater than the total amount of money received by the generators and the transmission owner, that is the following expression may not be equal to zero :

$p_{A} q_{A}+p_{B} q_{B}-\sum_{i=1}^{n_{A}} p_{A} q_{A}^{i}-\sum_{i=1}^{n_{B}} p_{B} q_{B}^{i}-b_{T O}\left|\sum_{i=1}^{n_{A}} q_{A}^{i}-q_{A}\right|$

In such a case, we assume that this quantity is kept by the ISO or an independent organization (not involved in forprofit market activities). The total amount of money collected during the whole period could be set apart to cover some of the maintenance costs of the system.

\section{The bidding strategy of active market participants}

The goal of each active market participant is to submit a bid that will maximize its payoff.

We assume that each active market participant knows the algorithm used by the ISO to solve the market as well as the mathematical formulation of the nodal price calculation.

The assumed behavior model for each market participant is based also on the following assumptions:

- each agent knows the load value $\left(q_{A}\right.$ and $\left.q_{B}\right)$;

- each agent knows the generation or transmission capacity of any of its competitors $\left(q_{A_{\max }}^{i}\right.$ and $\left.q_{B_{\max }}^{j} \forall i, j ; K\right)$;

- each agent knows the competitor bids at the previous stage $\left(b_{A_{t-1}}^{i}\right.$ and $\left.b_{B_{t-1}}^{j} \forall i, j ; b_{T O_{t-1}}\right)$;

Let us suppose that $B_{A}^{i}\left(B_{B}^{i}\right)$ is the set of possible bids for generator gen $_{A}^{i}\left(\right.$ gen $\left._{B}^{i}\right)$ and that $b_{A_{t}}^{i}\left(b_{B_{t}}^{i}\right)$ is the bid submitted by $g e n_{A}^{i}\left(\operatorname{gen}_{B}^{i}\right)$ at time $t^{2}$. Let $B_{T O}$ be the set of possible bids for the transmission owner and $b_{T O_{t}}$ the bid submitted by the $T O$ at time $t^{3}$.

We suppose that each agent submits the bid that would maximize its payoff if the other agents keep the same bids as the ones they submitted at the previous stage. By noticing that the value of the rewards of the different agents are functions of the bid submitted, the active agents compute therefore their bid as follows:

\footnotetext{
${ }^{2}$ The possible bids for the generators range between the marginal production cost and the price cap.

${ }^{3}$ The possible bids for the transmission owner range between the marginal transmission cost (equal to zero) and the price cap.
} 


$$
\begin{array}{cc}
b_{A_{t}}^{i}=\underset{b_{A}^{i} \in B_{A}^{i}}{\arg \max } & r_{g e n_{A}^{i}}\left(b_{A_{t-1}}^{1}, \cdots, b_{A}^{i}, \cdots, b_{A_{t-1}}^{n_{A}},\right. \\
b_{B_{t}}^{i}=\underset{b_{B}^{i} \in B_{B}^{i}}{\arg \max } & \left.b_{B_{t-1}}^{1}, \cdots, b_{B_{t-1}}^{n_{B}}, b_{T O_{t-1}}\right) \\
& r_{g e n_{B}^{i}}\left(b_{A_{t-1}}^{1}, \cdots, b_{A_{t-1}}^{n_{A}},\right. \\
b_{T O_{t}}=\underset{b_{T O} \in B_{T O}}{\arg \max } & \left.b_{B_{t-1}}^{1}, \cdots, b_{B}^{i}, \cdots, b_{B_{t-1}}^{n_{B}}, b_{T O_{t-1}}\right) \\
& r_{T O}\left(b_{A_{t-1}}^{1}, \cdots, b_{A_{t-1}}^{n_{A}},\right. \\
& \left.b_{B_{t-1}}^{1}, \cdots, b_{B_{t-1}}^{n_{B}}, b_{T O}\right)
\end{array}
$$

These three equations show that each active market participant solves several optimization problems to determine the expected payoff that could be obtained for each one of the possible bid strategies when the expected bids of the competitors are kept unchanged. Eventually, the chosen bid corresponds to the one that gives rise to the maximum expected payoff.

\section{Equilibrium}

The system is said to be in an equilibrium at time $t$ if $b_{A_{t+1}}^{i}=b_{A_{t}}^{i}, b_{B_{t+1}}^{j}=b_{B_{t}}^{j} \forall i \in\left\{1, \cdots, n_{A}\right\} \forall j \in$ $\left\{1, \cdots, n_{B}\right\}$ and $b_{T O_{t+1}}=b_{T O_{t}}$. Note that when a system is in an equilibrium point, an active market participant cannot increase its payoff by being the only one to change its bid function.

\section{Simulation CONDitions}

In the next two sections, we are going to simulate the market dynamics. We describe below the simulation conditions used. - The simulations are referred to a temporal horizon of 25 periods (number of stages). Therefore, the market dynamics is going to be integrated over 25 stages.

- The value of both loads $\left(q_{A}, q_{B}\right)$ is assumed constant and equal to $50 \mathrm{MW}$.

- The maximum admissible bid price (Price Cap) is equal to $4 \$ \mathrm{MW}^{4}$.

- The set of possible bid prices ( $B_{A}^{i}$ and $B_{B}^{j} \forall i, j$ and $B_{T O}$ ) is built by considering only the bids in the admissible range which are equal to (marginal_cost $+k * 0.1$ ) $\$$ /MW, where $k$ is an integer.

- When Eqns (13), (14) and (15) have multiple solutions (i.e. several bid prices give the same reward value), we suppose that the bid price submitted is the least expensive one.

- We suppose that at stage $t=0$ each agent assumes the bid of its competitors at the previous stage $(t=-1)$ to be equal to their marginal costs. In particular: $b_{A_{(-1)}}^{i}=c_{A}^{i} \forall i$ and $b_{B_{(-1)}}^{j}=c_{B}^{j} \forall j$ and $b_{T_{(-1)}}=0$.

\section{TWO GENERATORS IN THE SYSTEM}

\section{A. Description of the system}

The system we consider here has one generator connected to the left side and another generator connected to the right side $\left(n_{A}=n_{B}=1\right)$. We suppose that each generator can produce $100 \mathrm{MW}\left(q_{A_{\max }}^{1}=q_{B_{\max }}^{1}=100\right)$. Furthermore, we assume that $g e n_{A}^{1}$ has a marginal cost of $2 \$ / \mathrm{MW}\left(c_{A}^{1}=2\right)$ and $g e n_{B}^{1}$ a marginal cost of $3 \$ / \mathrm{MW}\left(c_{B}^{1}=3\right)$. We study in the next two paragraphs the market dynamics for two different values of the line transfer capacity.

${ }^{4}$ To be more accurate we should use as a unit of price $\$ /\left(\mathrm{MW}^{*}\right.$ duration of a stage). However to lighten the notations we simply use $\$ / M W$.

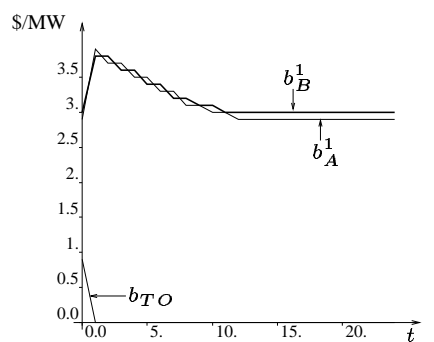

Fig. 2. Input of the market : the bids of $g e n_{A}^{1}, g e n_{B}^{2}$ and $T O$

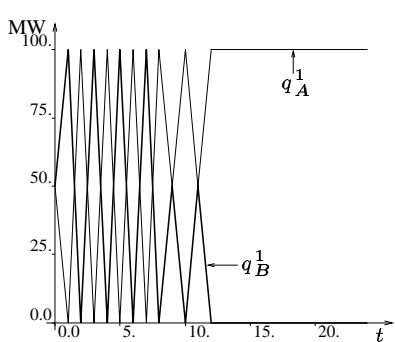

Production of $g e n_{A}^{\mathbf{1}}$ and $g e n_{B}^{\mathbf{1}}$

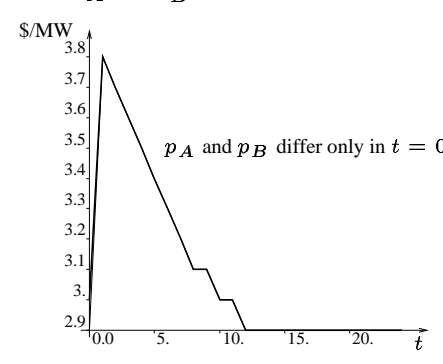

(b) Prices at node A and node B
Fig. 3. Output of the market : production of each generator and nodal prices

\section{B. Market dynamics: Transmission capacity of $50 \mathrm{MW}$}

This simulation refers to the case where the line transfer capacity is equal to $50 \mathrm{MW}$.

Figure 2 illustrates the bid strategy of each active market participant. The bids are calculated according to Eqns (13), (14) and (15).

The first offer of $T O$ is just slightly below the difference between the marginal costs of the two generators. However, due to the generators' bid strategies, the $T O$ bids are equal to zero in the next stages. Each generator tries to exploit its market power with its bids at the first stage. However, in the subsequent stages, the competition between the two power producers leads to a progressive undercutting of the bids. The process ends when the expensive generator gen $_{B}^{1}$ reaches its marginal cost. At that stage, an equilibrium point is reached. The transmission bid $\left(b_{T O}\right)$ is equal to zero, the $\operatorname{gen}_{B}^{1}$ bid is equal to its marginal costs $(3 \$ / \mathrm{MW})$ and the $g e n_{A}^{1}$ bid is slightly below the competitor bid, (namely (2.9 \$/MW).

In Figure 3 the quantity produced by each generator and the nodal prices are shown.

It is interesting to notice that in the first stage, when the transmission bid is different from zero, both the generators produce. Whereas, after that, until equilibrium is reached, the generator that submits the cheaper bid produces the total amount of power requested by the load.

The nodal prices at the two buses are the same at each stage, except for the first one when the prices are $p_{A}=2.9 \$ / \mathrm{MW}$ and $p_{B}=3 \$ / \mathrm{MW}$. It should be noted that after the first peak, the nodal prices decrease and reach their lowest values at the equilibrium. This value corresponds to $2.9 \$ / \mathrm{MW}$, namely the bid price of the cheapest generator $\left(\operatorname{gen}_{A}^{1}\right)$.

\section{Market dynamics: Transmission capacity of $25 \mathrm{MW}$}

In the following simulation the line transfer capacity is chosen to be $25 \mathrm{MW}$.

In this case the strategy is heavily influenced by the possible presence of congestion on the line. In Figure 4 the bidding 


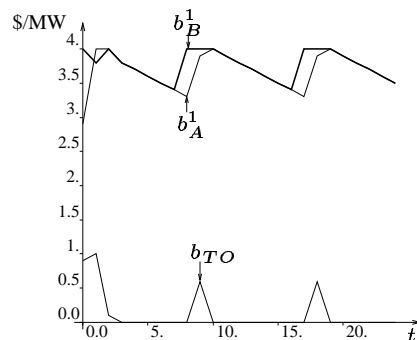

Fig. 4. Input of the market : the bids of $g e n_{A}^{1}, g e n_{B}^{2}$ and $T O$

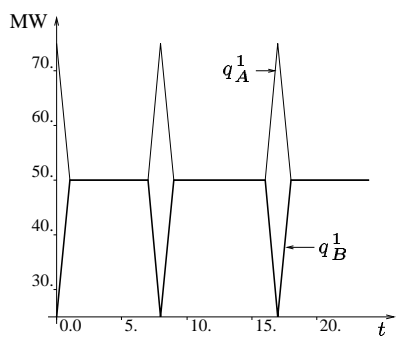

(a) Production of $g e n_{A}^{1}$ and $g e n_{B}^{1}$

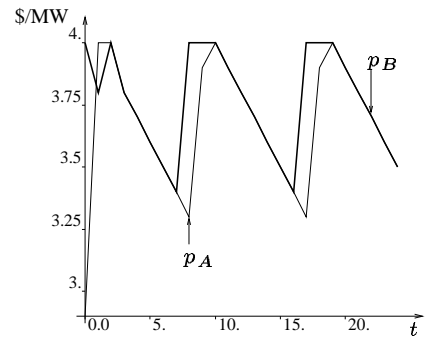

(b) Prices at node $\mathrm{A}$ and node $\mathrm{B}$
Fig. 5. Output of the market : production of each generator and nodal prices

strategies of the active players are shown. Figures IV-Ca-b show the production quantities and the nodal prices, respectively.

Since the capacity of the line is limited, neither generator can serve both loads. Therefore, both generators know that they will be dispatched for a minimum quantity of $25 \mathrm{MW}$, because of the limited capacity of the line $(K=25 \mathrm{MW})$ and the inelastic demand of $50 \mathrm{MW}$ at both buses.

In the following we discuss some features of the participant strategic behaviors and the market outcomes.

At the first stage, the cheapest generator available in the system $g e n_{A}^{1}$ offers a price just below the sum of the marginal cost of the expensive generator $\operatorname{gen}_{B}^{1}$ and the marginal cost of $T O$, since it assumes that both the competitors will bid at marginal cost. At the same time $g e n_{B}^{1}$ bids at the price cap, assuming that it will not be dispatched more than $25 \mathrm{MW}$, because its marginal cost is greater of the sum of the marginal costs of the rivals. The $T O$ bids just below the difference of the marginal costs of the power producers.

At the second stage $g e n_{A}^{1}$ changes its offer and bids at the price cap. It believes that by changing its bid to $4 \$ / \mathrm{MW}$ it will produce less $(50 \mathrm{MW})$ but at a much higher price and get in the end a higher reward. Generator gen $_{B}^{1}$ reduces its bid to $3.8 \$ / \mathrm{MW}$ to be less expensive than the bid of $g e n_{A}^{1}$ at the first stage plus the bid of $b_{T O}$ in order to be competitive with $g e n_{A}^{1}$ and cover the load at its bus. The $T O$ bids a price slightly below the difference between the competitors at the previous stage.

The results at this stage show that both generators are dispatched for the same quantity, both nodal prices are equal to the bid energy price at the bus, and no flow is observed in the line. Basically, the high transmission price prevented greater production from the generator with the lower bid $\left(\right.$ gen $\left._{B}^{1}\right)$.

At the third stage both generators bid at the price cap, assuming that the $T O$ continues to offer a very high transmission price. However, the $T O$ reduces its offer which becomes

\begin{tabular}{|c|c|c|c|c|c|c|}
\cline { 2 - 7 } \multicolumn{1}{c|}{} & \multicolumn{6}{c|}{$\begin{array}{c}\text { Rewards obtained by the different agents } \\
\text { of the system over } 25 \text { stages }\end{array}$} \\
\hline$K$ & gen $_{A}^{1}$ & gen $_{B}^{1}$ & $T O$ & $I S O$ & load $A$ & load $B$ \\
\hline 25 & 2,172 & 885 & 22.5 & 40 & $-4,585$ & $-4,710$ \\
\hline 50 & 1,980 & 205 & 0 & 0 & $-3,865$ & $-3,870$ \\
\hline
\end{tabular}

TABLE I

INFLUENCE OF LINE TRANSFER CAPACITY ON THE REWARDS.

less than the difference between the competitors' bids at the previous stage. The same observations made in the previous stage about the nodal prices and the dispatched quantities hold.

At the fourth stage, the TO bids at zero, because the difference between the expected competitors' bids is very small. Both the generators undercut their prices. Each one offers a bid price lower that the expected sum of the competitors' bids. The generators end up offering the same price, and consequently they are dispatched for the same quantity, and the nodal prices are equal to their bids.

In the following stages, we can observe a progressive undercutting, of the generators' bids until the more expensive generator reaches the value of 3.4 \$/MW. During all stages when the undercut strategy is adopted by the generators, the $T O$ continues to offer a zero transmission price. It knows that it has no chance to gain anything. The market outcomes show that each generator produces $50 \mathrm{MW}$, and receives its bid price. In fact, by comparing Figures 4 and b, it can be noted that the nodal price behaviors follow the generator bid behaviors exactly.

Any price lower than $3.4 \$ \mathrm{MW}$ implies a lower payoff for $\operatorname{gen}_{B}^{1}$ than the one obtained by bidding the price cap. During the stage when gen $_{B}^{1}$ offers the price cap, it is possible to observe a spike in the production of both generators. It corresponds to a production of $75 \mathrm{MW}$ by $\operatorname{gen}_{A}^{1}$ and $25 \mathrm{MW}$ by $g e n_{B}^{1}$. After this stage, both the $T O$ and $g e n_{A}^{1}$ raise their offers. Several cycles of undercut strategy can be observed in the aftermath. Each of them includes 9 stages. It can be noted that in the whole process no equilibrium point is reached.

\section{Market participant rewards (2 generators)}

In Table I the rewards of the active market agents are shown. The reward of the $I S O$ is also reported. This last quantity is calculated according to expression (12).

When the capacity of the line is limited, the transmission owner is able to gain a small amount of money. A limited capacity also favors the most expensive unit $\left(\operatorname{gen}_{B}^{1}\right)$ while gen $_{A}^{1}$ and the two loads benefit from a larger transfer capacity.

\section{FIVE GENERATORS IN THE SYSTEM}

\section{A. Description of the system}

The system we consider now is composed of 5 generators. Two machines are connected to node $A$ and three machines to node $B\left(n_{A}=2, n_{B}=3\right)$. The machines at bus $A$ have a lower marginal cost than the machines at bus $B$.

The characteristics of the generators are reported in Table II.

While in the previous section, one machine could generate, when the capacity of the line was enough, the energy for all the system it is not the case anymore here. And even together 


\begin{tabular}{|c|c|c|c|c|c|}
\cline { 2 - 6 } \multicolumn{1}{c|}{} & $\operatorname{gen}_{A}^{1}$ & gen $_{A}^{2}$ & gen $_{B}^{1}$ & gen $_{B}^{2}$ & gen $_{B}^{3}$ \\
\hline Marginal Cost $([\$ / \mathrm{MW}])$ & 2 & 2 & 3 & 3 & 3.5 \\
\hline Max. Capacity $([\mathrm{MW}])$ & 40 & 45 & 15 & 15 & 35 \\
\hline
\end{tabular}

TABLE II

CHARACTERISTICS OF THE GENERATORS.

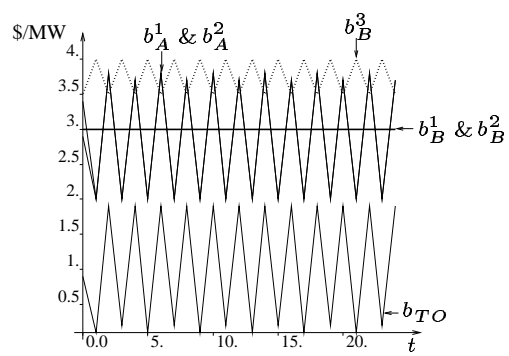

Fig. 6. Input of the market : the bids of the five generators and the $T O$

the machines located on one side could not generate all the power for the system.

Two cases are studied in the following. Each one refers to this system with a different transmission capacity.

\section{B. Market dynamics: Transmission capacity of $50 \mathrm{MW}$}

A transmission capacity of $50 \mathrm{MW}$ is considered hereafter.

In Figure 6 the competitors' bidding behavior is shown. In Figures $7 \mathrm{a}-\mathrm{b}$ the production quantities and the nodal prices are reported.

It can also be noted that in this case no equilibrium is reached during the whole period. Furthermore, without taking into account a short initial transient (first stage), a cyclic behavior can be observed for the bid strategies and therefore also for the market output. Each cycle goes on for 4 stages.

By observing Figure 6 , it can be noted that at node $A$, during the first half of the cycle, the bids of $g e n_{A}^{1}$ and $g e n_{A}^{2}$ swing between marginal costs $2 \$ / \mathrm{MW}$ and $3.8 \$ / \mathrm{MW}$. At the same time the $T O$ bids are $0.1 \$ / \mathrm{MW}$ and $1.9 \$ / \mathrm{MW}$, respectively. The second part of the cycle is characterized by $b_{A}^{1}$ and $b_{A}^{2}$ at $2 \$ / \mathrm{MW}$ when the $T O$ bid is $0.2 \$ / \mathrm{MW}$ and $b_{A}^{1}$ and $b_{A}^{2}$ equal to $3.7 \$ / \mathrm{MW}$ when $b_{T O}$ are equal to $1.9 \$ / \mathrm{MW}$. At node $B, \operatorname{gen}_{B}^{3}$ bids a price equal to the price cap (4 $\$ / \mathrm{MW}$ ) or equal to its marginal cost $(3.5 \$ / \mathrm{MW})$, depending on what it believes about the $T O$ and about $g e n_{A}^{i}$ behaviors. If the sum of the expected $b_{T O}$ and $\max \left(b_{A}^{1}, b_{A}^{2}\right)$ is lower than the $g e n_{B}^{3}$ marginal cost, it bids its marginal cost, whereas if the sum is greater than the price cap it offers the price cap. Generators $g e n_{B}^{1}$ and $g e n_{B}^{2}$ always offer their marginal cost, because they rely on the gen $_{B}^{3}$ bid to determine the nodal price at their bus.

These strategic bid behaviors lead to a maximum energy bid price at bus $A$ of one of these three values: $2,3.7$ or 3.8 $\$ / \mathrm{MW}$. When the bids $b_{A}^{1}$ and $b_{A}^{2}$ are equal to the generators' marginal costs (2 $\$ / \mathrm{MW})$, the transmission bid is also low. Therefore, the generators at bus $A$ produce at their maximum capacity $\left(q_{A}^{i}=q_{A_{\text {max }}}^{i} \forall i \in\left\{1, \ldots, n_{A}\right\}\right)$, as we can observe on Figure $7 \mathrm{a}$. When the energy bid prices $b_{A}^{1}$ and $b_{A}^{2}$ are higher than the generator marginal costs $c_{A}^{1}$ and $c_{A}^{2}$, the transmission bid price becomes to be so high that a dispatch with a flow from bus $B$ to bus $A$ is not convenient. In such a case, the sum of the dispatched quantity for the generators at bus $A$ is equal to $50 \mathrm{MW}$, the power consumed at node $\mathrm{A}$. The $p_{A}$

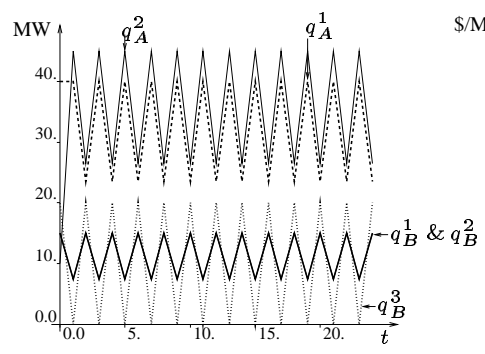

(a) Production of the five generators

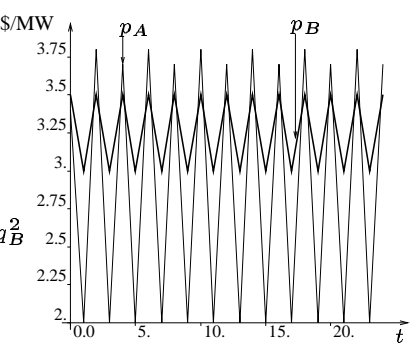

(b) Prices at node A and node $\mathrm{B}$

Fig. 7. Output of the market : production of each generator and nodal prices

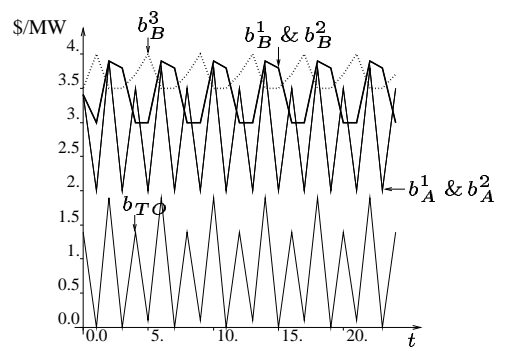

Fig. 8. Input of the market : the bids of the 5 generators and the TO

behavior corresponds to the energy bid price at that bus at each stage (Figure 7b). At bus $B$, gen $_{B}^{1}$ and $\operatorname{gen}_{B}^{2}$ cannot cover the whole load at that bus. Some power from gen ${ }_{B}^{3}$ or from bus $A$ is always needed. The choice about dispatching $g e n_{A}^{i}$ or $g e n_{B}^{3}$ and about what quantity $g e n_{B}^{1}$ and $g e n_{B}^{2}$ should produce is based on the comparison of two terms: (i) the sum of the energy bid price at bus $A$ and the transmission price and (ii) the bid price of $g e n_{B}^{3}$. These terms are alternately greater than the other. Therefore, the dispatched quantities swing for each generator between a minimum and a maximum value (Fig. 7a). The $p_{B}$ behavior always corresponds to the maximum energy bid price at bus $B$, among the accepted bids. It oscillates between the marginal costs 3 and 3.5 \$/MW (Fig. 7b).

It is interesting to notice, from the comparison between the values of $p_{A}$ and $p_{B}$, that the highest nodal price is experienced at bus $A$, where the production cost is lower. However, by comparing the average values of the two prices, it is clear that the higher mean value is experienced at bus $B$.

\section{Market dynamics: Transmission capacity of $25 \mathrm{MW}$}

In the following, we consider a line transfer capacity of $25 \mathrm{MW}$. The system considered is the same here as in the previous example, and is composed of five generators.

As in the previous case, no equilibrium point is reached. Except for the initial transient (3 stages), a cyclic behavior for all the bid prices can be observed (Figure 8). This type of strategic bids leads to an analogous cyclic behavior both for the production quantities and for the nodal prices, as shown in Figures 9a-b.

In this case, the strategic behavior of the active market participant is further complicated by the presence of the limited transmission capacity.

While the bid strategies of the generators at bus $A$ and the $T O$ are very similar to those in the previous case $(K=50$ MW), the strategy of the generators at bus $B$ is substantially different. In fact, we can observe that the competition among these three generators strongly influences their strategic bids. 

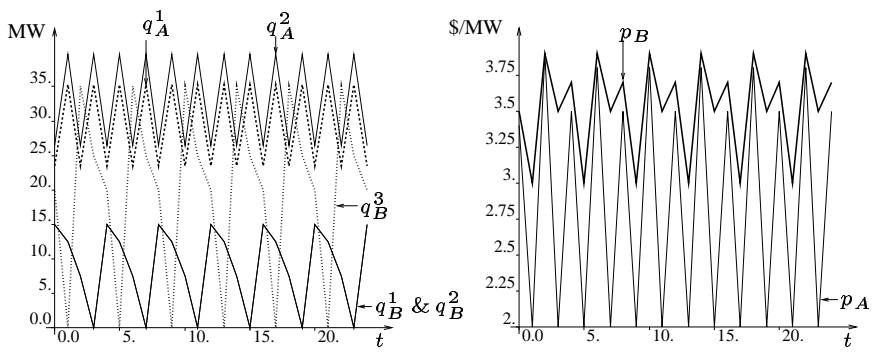

Production of the 5 generators

(b) Prices at node A and node B

Fig. 9. Output of the market : production of each generator and nodal prices

\begin{tabular}{|c|c|c|c|c|c|c|c|c|c|}
\cline { 2 - 10 } \multicolumn{1}{c|}{} & \multicolumn{8}{c|}{$\begin{array}{c}\text { Rewards obtained by the different agents } \\
\text { of the system over } 25 \text { stages }\end{array}$} \\
\hline$K$ & gen $_{A}^{1}$ & gen $_{A}^{2}$ & gen $_{B}^{1}$ & gen $_{B}^{2}$ & gen $_{B}^{3}$ & TO & ISO & load $A$ & load $B$ \\
\hline 25 & 501.2 & 563.8 & 111 & 111 & 108 & 12.5 & 362.5 & $-3,565$ & $-4,405$ \\
\hline 50 & 550.1 & 569.9 & 97.5 & 97.5 & 0 & 63 & 357 & $-3,620$ & $-4,075$ \\
\hline
\end{tabular}

TABLE III

INFLUENCE OF THE LINE TRANSFER CAPACITY ON THE REWARDS

\section{Market participant rewards (5 generators)}

The market participant rewards for the two cases previously analyzed are reported in Table III.

It interesting to notice that all the generators experience a variation in their payoffs with an increase in line transfer capacity. Some of them $\left(g e n_{A}^{1}, g e n_{A}^{2}\right)$ benefit from this increase, while others experience a decrease in their benefits $\left(\operatorname{gen}_{B}^{1}, \operatorname{gen}_{B}^{2}, \operatorname{gen}_{B}^{3}\right)$. However, the total amount of money paid by the loads is less in the case of higher transmission capacity. The money collected by the ISO decreases slightly when the maximum transmission capacity changes from 25 MW to $50 \mathrm{MW}$. The $T O$, on the other hand, experiences a significant increase in its sum of rewards when the line transfer capacity increases.

\section{MARKET TYPE AND REWARDS}

We have analyzed in these last two last sections the different rewards that the agents obtain in a market structure which allows the transmission owner to be an active market participant. In reference [5], the same study has been done for another type of market structure, namely a market working with the usual locational marginal price and without an active transmission owner. We propose now to compare the different rewards obtained by the actors for these two types of markets. In order to facilitate the comparison, all the relevant results have been gathered in Tables IV and V.

We can see by observing these two tables that the market structure strongly influences the rewards of the different agents.

\begin{tabular}{|c|c|c|c|c|c|c|}
\hline$K$ & gen $_{A}^{1}$ & gen $_{B}^{1}$ & TO & ISO & load $A$ & load B \\
\hline \multicolumn{7}{|c|}{ Location marginal price } \\
\hline 25 & $2,182.5$ & 715 & $/$ & 132.5 & $-4,465$ & $-4,640$ \\
\hline 50 & 2,250 & 0 & $/$ & 0 & $-3,625$ & $-3,625$ \\
\hline \multicolumn{7}{|c|}{ Active transmission owner } \\
\hline 25 & 2,172 & 885 & 22.5 & 40 & $-4,585$ & $-4,710$ \\
\hline 50 & 1,980 & 205 & 0 & 0 & $-3,865$ & $-3,870$ \\
\hline
\end{tabular}

TABLE IV

REWARDS AND MARKET TYPE. THE TWO MACHINE POWER SYSTEM

\begin{tabular}{|c|c|c|c|c|c|c|c|c|c|}
\hline$K$ & gen $_{A}^{1}$ & gen $_{A}^{2}$ & gen $_{B}^{1}$ & gen $_{B}^{2}$ & gen $_{B}^{3}$ & TO & ISO & load A & load B \\
\hline \multicolumn{10}{|c|}{ Locational marginal price } \\
\hline 25 & 633 & 712 & 68 & 68 & 0 & $/$ & 300 & -3410 & -4010 \\
\hline 50 & 1000 & 1125 & 0 & 0 & 0 & $/$ & 0 & -3750 & -3750 \\
\hline \multicolumn{10}{|c|}{ Active transmission owner } \\
\hline 25 & 501.2 & 563.8 & 111 & 111 & 108 & 12.5 & 362.5 & $-3,565$ & $-4,405$ \\
\hline 50 & 550.1 & 569.9 & 97.5 & 97.5 & 0 & 63 & 357 & $-3,620$ & $-4,075$ \\
\hline
\end{tabular}

TABLE V

REWARDS AND MARKET TYPE. THE FIVE MACHINE POWER SYSTEM.

It may be surprising to note that machines located on the left side always earn less when dealing with an "Active transmission owner" market type. These machines have lower marginal costs than machines located on the right side. And although in the "Locational marginal price" market they could bid strategically against the other machines to make more money, it is slightly different here. They enter in competition with this new actor, which decreases the amount of money they can make exporting power to the right side.

Another interesting remark suggested by the analysis of these two tables is that the idea of an active transmission owner tends to favor the agents responsible for the transmission (the couple TO-ISO). Notice that with the market structure introduced in this paper, the two actors can make money even when no congestion occurs. Note also that if the couple TOISO earns more in this market structure, the load seems to be penalized.

Although this analysis is certainly not sufficient to be able to conclude which market structure could be more efficient in terms of competitiveness, it shows however that the approach we have adopted to evaluate the different payoffs of the agents may be used to design some market rules.

\section{CONCLUSIONS}

We have considered in this paper a new type of market in which the transmission owners offer at each market stage a bid for their services. To study this type of market, we have modelled the behavior of the different agents of the system and created a market dynamics. By integrating this market dynamics over a certain temporal horizon we have been able to get some insights into the complex phenomena that may occur in this electricity market, to assess the payoffs of the different agents (loads, power producers, transmission owners, ISO) of the system and to determine the influence of the available transmission capacities on these payoffs. Since the approach we have used to model the agents' behavior can be used with other market types, we have also been able to illustrate how the different agent payoffs are influenced by the market rules.

\section{REFERENCES}

[1] W. Hogan, "Transmission Market Design," JFK School of Government, Harvard Electricity Policy Group Harvard University, Tech. Rep., 2003.

[2] M. Barmack, P. Griffes, E. Kahn, and S. Oren, 'Performance incentives for transmission," The Electricity Journal, vol. 16, no. 3, pp. 9-22, April 2003.

[3] W. Hogan, 'Financial Transmission Rights Formulation," JFK School of Government, Harvard Electricity Policy Group Harvard University, Tech. Rep., 2002.

[4] I. Vogelsang, 'Price Regulation for Independent Transmission Companies," Journal of Regulatory Economics, vol. 20, no. 2, pp. 141-165, 2001.

[5] D. Ernst, A. Minoia, and M. Ilic, "Market dynamics driven by the decision-making power producers," Submitted to IEEE PES 2004 Meeting. A draft is available at http://www.montefi ore.ulg.ac.be/ ernst/, 2003. 